\title{
STRONGLY LOCALLY SETWISE HOMOGENEOUS CONTINUA AND THEIR HOMEOMORPHISM GROUPS $\left(^{1}\right)$
}

\author{
BY \\ BEVERLY L. BRECHNER( $\left.{ }^{2}\right)$
}

0. Introduction. In this paper we define strongly locally setwise homogeneous continua (s-l-s-h continua) and determine the minimal normal subgroups of the groups of all homeomorphisms of such continua. We also obtain some results about the topological structure of such spaces. Locally setwise homogeneous (l-s-h) continua were first introduced in [1], where it is shown that the groups of all homeomorphisms of l-s-h continua are nonzero dimensional. These continua include all the compact, connected manifolds, with or without boundary, as well as the universal plane curve (i.e. Sierpiński curve), universal curve, and Hilbert cube. These examples are also s-l-s-h continua except possibly the universal curve and Hilbert cube.

In [3] R. D. Anderson determines the minimal normal subgroups of the groups of all homeomorphisms of certain topological spaces with a complicated kind of setwise homogeneity and an invertibility property. These spaces include the universal plane curve, universal curve, $S^{2}$, and $S^{3}$.

In [2] J. V. Whittaker shows that the compact manifolds, with or without boundary, are characterized by the algebraic structure of their groups of homeomorphisms, in the sense that any two compact manifolds are homeomorphic iff their groups of homeomorphisms are algebraically isomorphic. One of his main tools in this characterization is his development, in the first part of the paper, of the structure of the minimal normal subgroups of the groups of all homeomorphisms of the compact manifolds. His methods are similar to those of [3].

In [7] Ulam and von Neumann announce the theorem that the identity component of the homeomorphism group of the two-sphere is simple.

Thus our results of $\S 3$ will extend some of the results of [2], [3], and [7]. The paper is organized as follows. In $\$ 2$, we develop the notion of a near basis for a s-1-s-h continuum; in $\S 3$, we use near bases to determine the minimal normal subgroups of the groups of homeomorphisms of s-1-s-h continua; in $\$ 4$, we study the structure of s-l-s-h continua some more; and finally in $\$ 5$, we show that various

Received by the editors October 31, 1968 and, in revised form, February 27, 1970.

(1) This paper is a correction of a paper presented to the Society on January 23, 1969 under the title Locally setwise homogeneous continua and their homeomorphism groups.

$\left({ }^{2}\right)$ The author would like to thank the referee for many helpful comments and for his simplified proof of Corollary 3.1.1. 
continua are both 1-s-h and also s-l-s-h. We also show that the Hilbert cube is $1-\mathrm{s}-\mathrm{h}$.

The following important question remains unanswered: Are all l-s-h continua also s-1-s-h?

1. Conventions, notation, and some definitions. All spaces are separable metric. $H(X)$, or $H$ when no confusion arises, denotes the group of all homeomorphisms of $X$.

$f: X \rightarrow Y$ denotes an onto function.

If $A \subseteq X$, then $h$ is supported on $A$ means that $h(x)=(x)$ for $x \notin A$.

For each point $x \in X, O(x)$ denotes the orbit of $x$; that is, $O(x)=\{h(x) \mid h \in H\}$.

A chain of open sets $\mathscr{U}: U_{1}, \ldots, U_{n}$ is a finite, ordered set of open sets such that

(1) $U_{i} \cap U_{j} \neq \varnothing$ iff $|i-j| \leqq 1$,

(2) $\bar{U}_{i} \cap \bar{U}_{j} \neq \varnothing$ iff $U_{i} \cap U_{j} \neq \varnothing$, and

(3) $\bar{U}_{i} \neq \bar{U}_{j}$ for any pair $i, j$.

$\mathscr{U}$ is a chain from $a$ to $b$ means that $a$ is an element only of the first link of $\mathscr{U}$, and $b$ is the element only of the last link of $\mathscr{U}$, and neither is contained in the closure of the remaining elements of $\mathscr{U}$. It is well known and easy to prove that if $a$ and $b$ are points of a connected, locally connected, metric space, then for any $\varepsilon>0$, there is a chain from $a$ to $b$ such that the diameter of each link is less than $\varepsilon$. Furthermore, these elements may be chosen from any basis for the space.

If $\mathscr{U}$ is a collection of sets, then $\mathscr{U}^{*}$ denotes the union of the elements of $\mathscr{U}$. $\mu(\mathscr{U})=\operatorname{lub}\{\operatorname{diam} U \mid U \in \mathscr{U}\}$ is called the mesh of $\mathscr{U}$.

If $A \subseteq X, C(A)$ denotes the complement of $A . \bar{A}$ or $A^{-}$denotes closure.

The universal plane curve (or Sierpinski curve) is a continuum whose standard construction is the following: Let $C$ be a square plus its interior in the plane. Divide $C$ into nine equal squares, and remove the interior of the middle ninth. Break each of the remaining eight squares into nine equal squares, and remove the interiors of their middle ninths. Continue the process inductively, ad infinitum. The set which remains is the desired continuum. The Sierpiński curve has been characterized by Whyburn [6] as the only locally connected, one-dimensional plane continuum with no local cut points.

The standard construction for the universal curve (or Menger curve) is the following: Let $F_{1}, F_{2}$, and $F_{3}$ be three faces of the solid cube $S$ in $E^{3}$ such that no two of these faces are opposite each other. We remove portions of $S$ by punching out, to the opposite side, the interiors of the middle ninths of $F_{1}, F_{2}$, and $F_{3}$. We next punch out, to the opposite side, the interiors of the middle ninths of each of the remaining eight squares in each of $F_{1}, F_{2}$, and $F_{3}$, and we continue this process inductively. The remaining set is a continuum called the universal curve. The universal curve has been characterized by R. D. Anderson [4] as the only locally connected, one-dimensional metric continuum containing no local cut points, such that no open subset is imbeddable in the plane. 
2. Strongly locally setwise homogeneous continua and near bases. In this section we study the structure of s-l-s-h continua through the notion of a near basis for the space. We begin with some definitions.

Definition 2.1. Let $X$ be a continuum, and let $G$ be a subgroup of $H(X)$. Then $X$ is called locally setwise homogeneous under $G$ iff there exist both a basis $\mathfrak{B}$ of connected open sets of $X$ and a dense subset $A$ of $X$ such that for any $B \in \mathfrak{B}$ and $a, b \in A \cap B$, there is a homeomorphism $g \in G, g$ supported on $B$, such that $g(a)=b .\{X, A, \mathfrak{B}, G\}$ is called a locally setwise homogeneous structure for $X$. If $X$ is locally setwise homogeneous under $H(X)$, then $X$ is called locally setwise homogeneous denoted $1-\mathrm{s}-\mathrm{h}$. We note that a l-s-h continuum will necessarily be locally connected.

Definition 2.2. A continuum $X$ is called strongly locally setwise homogeneous (s-l-s-h) iff there exists a locally setwise homogeneous structure $\{X, A, \mathfrak{B}, G\}$ for $X$ such that for each $B \in \mathfrak{B}$ and $x \in A \cap B$, there is a neighborhood $U$ of $x$ with $\bar{U} \subseteq B$, satisfying the following property: For each open subset $V$ of $B$, there exists a homeomorphism $h \in G$ such that

(1) $h$ is supported on $B$, and

(2) $h(\bar{U}) \subseteq V$.

We will say that $U$ (or $\bar{U}$ ) moves freely in $B$.

REMARK. We note that, in this paper, l-s-h and s-l-s-h structures and spaces are defined only for locally connected continua, although it is possible to define them for more general spaces.

Definition 2.3. Let $\{X, A, \mathfrak{B}, H\}$ be a l-s-h structure, and let $B \in \mathfrak{B}, B \neq \varnothing$. Let $\subseteq=\{h(B) \mid h \in H\}$. $\subseteq$ is called a near basis for $X$ iff every open set $U$ of $X$ contains the closure of an element of $\mathfrak{S}$. $\subseteq$ is said to be generated by $B$.

See Remark after Theorem 2.2.

Lemma 2.1. Let $\{X, A, \mathfrak{B}, H\}$ be a s-l-s-h structure. Let $\mathscr{U}: U_{1}, U_{2}, \ldots, U_{n}$ be a chain of elements of $\mathfrak{B}$ from $x$ to $y$, where $x, y \in A$. Then there exists a neighborhood $N$ of $x$ such that $N \subseteq\left(U_{1}-\bar{U}_{2}\right)$ and $N$ moves freely in $\mathscr{U}^{*}$. Further there exists a homeomorphism $g$ such that $g=h_{n-1} \ldots h_{2} h_{1}$, where $h_{i}$ is supported on $U_{i}$ and $g(N)$ moves freely in $U_{n}$.

Proof. Since $X$ is s-l-s-h, there exists a neighborhood $N^{\prime}$ of $x$ in $U_{1}$ which moves freely in $U_{1}$. Let $d=d\left(x, \bar{U}_{2}\right)$. Let $N$ be a connected neighborhood of $x$ such that $N \subseteq N^{\prime}$ and $\operatorname{diam}(N)<d$. We show that $N$ moves freely in $\mathscr{U}^{*}$. Let $x_{1} \in U_{1} \cap U_{2} \cap A$. Then there exists a neighborhood $N_{1}$ of $x_{1}$ such that $N_{1} \subseteq U_{1} \cap U_{2}$ and $N_{1}$ moves freely in $U_{2}$. Since $N_{1}$ is open in $U_{1}$ and $N$ moves freely in $U_{1}$, there exists a homeomorphism $h_{1}$ supported on $U_{1}$ such that $h_{1}(\bar{N})$ $\subseteq N_{1}$. Now let $V$ be any open set in $U_{2}$. There is a homeomorphism $\phi_{1}$ supported on $U_{2}$ such that $\phi_{1}\left(\bar{N}_{1}\right) \subseteq V$. Thus $\phi_{1} h_{1}(\bar{N}) \subseteq V$. It follows that $N$ moves freely in $U_{1} \cup U_{2}$.

Now let $x_{2} \in U_{2} \cap U_{3}$ and let $N_{2}$ be a neighborhood of $x_{2}$ that moves freely in 
$U_{3}$. Then, since $N_{1}$ moves freely in $U_{2}$, there exists a homeomorphism $h_{2}$ supported on $U_{2}$ such that $h_{2}\left(\bar{N}_{1}\right) \subseteq N_{2}$. Therefore $h_{2} h_{1}(\bar{N}) \subseteq N_{2}$.

We may continue this process inductively. Thus, after a finite number of steps, we have neighborhoods $N_{i} \subseteq U_{i} \cap U_{i+1}$ for $1 \leqq i \leqq n-1$, such that

(1) $N_{i}$ moves freely in $U_{i+1}$, and

(2) $h_{i} \ldots h_{2} h_{1}(\bar{N}) \subseteq N_{i}$ and moves freely in $U_{i+1}$.

Now let $N_{n}$ be any open set in $U_{n}$. Since $h_{n-1} \ldots h_{2} h_{1}(\bar{N})$ moves freely in $U_{n}$, there exists a homeomorphism $h_{n}$ such that $h_{n} h_{n-1} \ldots h_{2} h_{1}(\bar{N}) \subseteq N_{n}$. Thus $N$ moves freely in $\mathscr{U}^{*}$, and if $g=h_{n-1} \ldots h_{1}$, then $g(N)$ moves freely in $U_{n}$.

Lemma 2.2. Let $\{X, A, \mathfrak{B}, H\}$ be a s-l-s-h structure. Then for each $x \in X$ and connected open set $U$ containing $x$, there exist a sequence of connected open sets $\left\{W_{i}\right\}_{i=0}^{\infty}$ in $U$ and a homeomorphism $h \in H, h$ supported on $U$, such that

(1) $W_{i} \cap W_{j}=\varnothing$ for $i \neq j$,

(2) $\lim _{i \rightarrow \infty} W_{i}=x$, and

(3) $h^{i+1}\left(W_{0}\right)=h\left(W_{i}\right)=W_{i+1}$.

Proof. Let $x \in X$ and let $U$ be a connected open set containing $x$. Let $a \in A \cap U$, $a \neq x$. Let $\mathscr{U}_{1}: U_{1,1}, U_{1,2}, \ldots, U_{1, n_{1}}$ be a chain of elements of $\mathfrak{B}$ in $U$ from $a$ to $x$ such that $\mu(\mathscr{U})<\frac{1}{3} d(a, x)$. Let $a_{1,0}=a, a_{1, i} \in A \cap\left(U_{1, i} \cap U_{1, i+1}\right)$ for $i=1,2, \ldots$, $n_{1}-1$. Let $\mathscr{U}_{2}: U_{2,1}, U_{2,2}, \ldots, U_{2, n_{2}}$ be a chain of elements of $\mathfrak{B}$ from $a_{1, n_{1}-1}$ to $x$ in $U_{1, n_{1}}$ such that $\mu\left(\mathscr{U}_{2}\right)<\frac{1}{3} d\left(\bar{U}_{1, n_{1}-1}, x\right)$. Let $a_{1, n_{1}-1}$ be $a_{2,0}$ and let $a_{2, i} \in A \cap\left(U_{2, i} \cap U_{2, i+1}\right)$ for $i=1,2, \ldots, n_{2}-1$. Let $\mathscr{U}_{3}: U_{3,1}, U_{3,2}, \ldots, U_{3, n_{3}}$ be a chain of elements of $\mathfrak{B}$ in $U_{2, n_{2}}$ from $a_{2, n_{2}-1}$ to $x$ such that $\mu\left(\mathscr{U}_{3}\right)<\frac{1}{3} d\left(\bar{U}_{2, n_{2}-1}, x\right)$. Clearly this process may be continued inductively, ad infinitum.

Now let $\mathscr{V}_{1}$ be the subchain of $\mathscr{U}_{1}$ whose elements are $U_{1,1}, U_{1,2}, \ldots, U_{1, n_{1}-1}$. Let $\mathscr{V}_{2}$ be the subchain of $\mathscr{U}_{2}$ from the last element of $\mathscr{U}_{2}$ which meets the last element of $\mathscr{V}_{1}$ to (and including) $U_{2, n_{2}-1}$. Let $\mathscr{V}_{3}$ be the subchain of $\mathscr{U}_{3}$ from the last element of $\mathscr{U}_{3}$ which meets $\mathscr{V}_{2}$ to $U_{3, n_{3}-1}$. Continue the process inductively. Let $\mathscr{V}$ be the infinite chain whose links are the links of the $\mathscr{V}_{i}^{\prime}$ 's. Now we rename the links of the chains $\mathscr{V}_{i}$ as follows:

$\mathscr{V}_{1}: V_{1,1}, V_{1,2}, \ldots, V_{1, k_{1}}$,

$\mathscr{V}_{2}: V_{2,1}, V_{2,2}, \ldots, V_{2, k_{2}}$, etc.

Let $b_{1,1} \in A \cap\left(V_{1,1}-\bar{V}_{1,2}\right), b_{1,2} \in A \cap\left(V_{1,1} \cap V_{1,2}\right), \ldots, b_{1, k_{1}} \in A \cap\left(V_{1, k_{1}-1}\right.$ $\left.\cap V_{1, k_{1}}\right)$. Let $b_{2,1} \in A \cap\left(V_{2,1}-\bar{V}_{2,2}\right) \cap V_{1, k_{1}}, b_{2,2} \in A \cap\left(V_{2,1} \cap V_{2,2}\right), \ldots$, $b_{2, k_{2}} \in A \cap\left(V_{2, k_{2}-1} \cap V_{2, k_{2}}\right)$. We continue this process inductively, also.

Finally we are ready to construct the homeomorphism $h$. Let $N_{1}$ be a connected neighborhood of $b_{1,1}$ in $V_{1,1}-\bar{V}_{1,2}$ which moves freely in $V_{1,1}$. Let $N_{2}$ be a neighborhood of $b_{2,1}$ in $V_{1, k} \cap V_{2,1}$ which moves freely in $V_{2,1}$. Let $M_{1}$ be a neighborhood of $b_{2,2}$ in $V_{2,1} \cap V_{2,2}$ which moves freely in $V_{2,2}$. We choose $N_{1}, N_{2}$, and $M_{1}$ so that they are elements of $\mathfrak{B}$. Let $g_{1}$ be a homeomorphism supported on $V_{2,1}$ such that $g_{1}\left(N_{2}\right) \subseteq M_{1}$. Let $\mathscr{W}_{1}$ be the chain $\mathscr{V}_{1}$, and let $h_{1}$ be a homeomorphism supported on $\mathscr{W}_{1}^{*}$ such that $h_{1}\left(N_{1}\right) \subseteq N_{2}$. We further assume that $h_{1}$ is constructed as $g$ 
is constructed in Lemma 2.1. Note that $h_{1} g_{1}$ is supported on $\mathscr{V}_{1}^{*} \cup V_{2,1}$ and takes $N_{1}$ into $N_{2}$ and $N_{2}$ into $M_{1}$.

Let $N_{3}$ be a neighborhood of $b_{3,1}$ in $V_{2, k_{2}} \cap V_{3,1}$ which moves freely in $V_{3,1}$. Let $M_{2}$ be a neighborhood of $b_{3,2}$ in $V_{3,1} \cap V_{3,2}$ which moves freely in $V_{3,2}$. Let $g_{2}$ be a homeomorphism supported on $V_{3,1}$ such that $g_{2}\left(N_{3}\right) \subseteq M_{2}$. Let $\mathscr{W}_{2}$ be the following chain: $\mathscr{W}_{2}: V_{2,2}, V_{2,3}, \ldots, V_{2, k_{2}}$. Then, as above, there exists a homeomorphism $h_{2}$ supported on $\mathscr{W}_{2}^{*}$ such that $h_{2}\left(M_{1}\right) \subseteq N_{3}$. Again we assume that $h_{2}$ is constructed as $g$ is constructed in Lemma 2.1. Note that neither $g_{2}$ nor $h_{2}$ affects points of $V_{1,1}, \ldots, V_{1, k_{1}-1}$. Further $h_{2} g_{2} h_{1} g_{1}$ is supported on $\mathscr{V}_{1}^{*} \cup \mathscr{V}_{2}^{*}$ and takes $N_{1}$ into $N_{2}, N_{2}$ into $N_{3}$ and $N_{3}$ into $M_{2}$. We continue the process inductively obtaining two sequences of homeomorphisms $\left\{g_{i}\right\}_{i=1}^{\infty}$ and $\left\{h_{i}\right\}_{i=1}^{\infty}$.

It is clear that $g_{1}, h_{1}$, and $h_{2}$ are the only homeomorphisms affecting points of $\mathscr{V}_{1}^{*}$. Similarly, $g_{n-1}, g_{n}, h_{n-1}, h_{n}$, and $h_{n+1}$ are the only homeomorphisms affecting points of $\mathscr{V}_{n}^{*}$. Thus we may define $h=\cdots h_{2} \cdot g_{2} \cdot h_{1} \cdot g_{1}=\prod_{i=1}^{\infty} h_{i} g_{i}$, since for each $n, \prod_{i=1}^{\infty} h_{i} g_{i}=\prod_{i=1}^{n+1} h_{i} g_{i}$ on $\mathscr{V}_{n}^{*}$. Then $h$ is clearly a homeomorphism of $X$ onto itself such that $h$ is supported on $\mathscr{V}^{*}$ and $h\left(N_{i}\right) \subseteq N_{i+1}$.

We obtain $\left\{W_{i}\right\}_{i=0}^{\infty}$ as follows. Let $W_{0}=N_{1}$ and let $W_{n}=h^{n}\left(N_{1}\right)$. Since $N_{1} \cap N_{2}$ $=\varnothing$ and $h\left(N_{1}\right) \subseteq N_{2}$, it follows that $W_{0} \cap W_{1}=\varnothing$. Therefore $h^{n}\left(W_{0}\right) \cap h^{m}\left(W_{0}\right)=\varnothing$ also. For suppose not, and assume $n>m$. Let $y \in h^{n}\left(W_{0}\right) \cap h^{m}\left(W_{0}\right)$. Then $h^{-m}(y)$ $\in W_{0} \cap h^{n-m}\left(W_{0}\right)$. But $n-m>0$ and $h^{k}\left(W_{0}\right)$ is a subset of $\bigcup_{i=2}^{\infty} \mathscr{V}_{i}^{*}$ for $k>1$. This is a contradiction. Thus $W_{i} \cap W_{j}=\varnothing$ for $i \neq j$, and (1) of the lemma is proved.

To show part (2) we observe that $N_{i} \subseteq V_{i-1, k_{i-1}} \cap V_{i, 1}, \lim _{i \rightarrow \infty}\left(\operatorname{diam} V_{i, 1}\right)=0$, and $\lim _{i \rightarrow \infty} d\left(V_{i-1, k_{i-1}}, x\right)=0$. Thus $\lim _{i \rightarrow \infty} N_{i}=x$. But $W_{i} \subseteq N_{i}$. It follows that $\lim _{i \rightarrow \infty} W_{i}=x$, and (2) is proved.

Part (3) follows immediately, because $W_{n}=h^{n}\left(W_{0}\right)$ by definition. Thus the lemma is proved.

REMARK. We note that the $N_{i}$ 's could have been chosen to be elements of $\mathfrak{B}$.

LemmA 2.3. Let $\{X, A, \mathfrak{B}, H\}$ be a s-l-s-h structure. Then the subspace $A$ admits a basis of homeomorphic sets.

Proof. Let $x_{0} \in A$ and let $\left\{W_{i}\right\}_{i=0}^{\infty}$ be a sequence of connected open sets such that

(1) $W_{i} \cap W_{j}=\varnothing$ for $i \neq j$,

(2) $\lim _{i \rightarrow \infty} W_{i}=x_{0}$, and

(3) $W_{n}=h^{n}\left(W_{0}\right)$ for some homeomorphism $h$, as in Lemma 2.2.

Let $x$ be any point of $A$ and let $U \in \mathfrak{B}$ be any neighborhood of $x$. We will show that there is a homeomorphism $\phi$ such that $x \in \phi\left(W_{0}\right) \subseteq U$.

Since $x \in A$, there exists a homeomorphism $g$ such that $g\left(x_{0}\right)=x$. Let $g\left(W_{i}\right)=W_{i}^{\prime}$. Then $W_{i}^{\prime} \rightarrow x$. Therefore there is an integer $n$ such that $W_{n}^{\prime} \subseteq U$. Let $y \in A \cap W_{n}^{\prime}$. Then there is a homeomorphism $f$, supported on $U$, such that $f(x)=y$. Therefore $f^{-1}(y)=x$ and $x \in f^{-1}\left(W_{n}^{\prime}\right) \subseteq U$. Let $\phi=f^{-1} g h^{n}$. Then $x \in \phi\left(W_{0}\right) \subseteq U$. It follows that $A$ admits a basis of homeomorphic sets-namely the collection of all images of $W_{0}$ under homeomorphisms of $X$ onto itself. 
THEOREM 2.1. Every s-l-s-h continuum has a near basis.

Proof. Let $\{X, A, \mathfrak{B}, H\}$ be a s-l-s-h structure, and let $x_{0} \in A$. Let $\left\{W_{i}\right\}_{i=0}^{\infty}$ be a sequence of pairwise disjoint elements of $\mathfrak{B}$ converging to $x_{0}$, such that for some $h \in H, h\left(W_{i}\right)=W_{i+1}$. Let $\mathfrak{E}=\left\{h\left(W_{0}\right) \mid h \in H\right\}$. Then it follows from Lemma 2.3 that $\mathbb{E}$ is a near basis for $X$, for if $U$ is any (connected) open set of $X$, there is a point $a \in A \cap U$, and therefore an element $E \in \mathbb{C}$ such that $\bar{E} \subseteq U$.

THEOREM 2.2. Let $X$ be a s-l-s-h continuum, and let $\mathfrak{S}_{1}$ and $\mathfrak{\Im}_{2}$ be two near bases for $X$, generated by $B_{1}$ and $B_{2}$, respectively. Let $M\left(\mathfrak{C}_{i}\right)=\bigcup\left\{E \in \mathfrak{C}_{i}\right\}, i=1,2$. Then $M\left(\mathfrak{C}_{1}\right)=M\left(\mathfrak{C}_{2}\right)$.

Proof. Let $x \in M\left(\mathfrak{C}_{1}\right)$. It is sufficient to show that $x \in M\left(\mathfrak{S}_{2}\right)$. Since $x \in M\left(\mathfrak{C}_{1}\right)$, there exists an $h \in H$ such that $x \in h\left(B_{1}\right)$. Now $B_{2}$ is open in $X$, and $\mathfrak{S}_{1}$ is a near basis for $X$. Therefore there is a homeomorphism $g \in H$ such that $g\left(B_{1}\right) \subseteq B_{2}$. Then $g h^{-1}$ takes $h\left(B_{1}\right)$ into $B_{2}$. It follows that $h g^{-1}\left(B_{2}\right)$ contains $x$ and is an element of $\mathfrak{C}_{2}$. Therefore $x \in M\left(\mathfrak{C}_{2}\right)$.

REMARK. It follows from Theorem 2.2 that $M(\mathbb{E})$ is independent of the near basis $\mathbb{E}$ chosen for $X$, and is therefore unique. Thus we may, and will, denote this set by $M(X)$, and call it the core of $X$. We note that in the case of the compact connected manifolds, this set coincides with the set $M(X)$ defined in [2], and is just the interior of the manifold. In the case of the universal plane curve, we get the whole space. This latter statement is true for the following reasons. It follows from [6] that the universal plane curve has exactly two orbits (one of which is the union of the complementary bounding simple closed curves), and each of these orbits is dense in the space. In Theorem 5.2, we prove that the universal plane curve is s-1-s-h. Thus we may apply Theorem 4.2, in which it is proved that the core of $X$ is the union of the dense orbits of $X$. Thus the core of the universal plane curve is the whole space.

3. Minimal normal subgroups. Theorem 3.1 of this section is an analogue of Theorem 1 of [2]. The proof is essentially the same. We will indicate how to use the properties of the previous section to make the necessary modifications.

Definition 3.1. Let $X$ be a s-l-s-h continuum, and let \& be a near basis for $X$. Let $Q(\mathbb{E})$ be the group of homeomorphisms generated by the homeomorphisms supported on elements of $\mathbb{E}$; that is, $Q(\mathbb{E})$ consists of finite products of homeomorphisms, each of which is supported on an element of $\mathbb{E}$. Then $Q(\mathfrak{E})$ is called a basic subgroup of $H(X)$ and is said to be generated by $\leftleftarrows$.

See Remark after Corollary 3.1.1.

THEOREM 3.1. Let $X$ be a s-l-s-h continuum, and let $Q(\mathbb{E})$ be a basic subgroup of $H(X)$ generated by the near basis $\mathfrak{E}$. Then $Q(\mathfrak{E})$ is a minimal normal subgroup of $H(X)$.

Proof. Let $\{X, A, \mathfrak{B}, H\}$ be a s-1-s-h structure. Let $G$ be any nontrivial normal subgroup of $H$, and let $g \in G, g \neq e$. We will show that $Q(\circledast) \subseteq G$. Let $U$ be any 
element of \& such that $U \cap g^{-1}(U)=\varnothing$. We note that $g^{-1}(U) \in \mathbb{E}$ also. Let $x$ be an element of $U$, and let $\left\{W_{i}\right\}_{i=0}^{\infty}$ be a sequence of connected open sets in $U$, and let $f_{1}$ be an element of $H$ such that

(1) $\lim _{i \rightarrow \infty} W_{i}=x$,

(2) $W_{i} \cap W_{j}=\varnothing$ for $i \neq j$, and

(3) $f_{1}\left(W_{i}\right)=W_{i+1}$.

By Lemma 2.2, $\left\{W_{i}\right\}_{i=0}^{\infty}$ and $f_{1}$ exist. Further $f_{1} \in Q(\mathfrak{E})$, since $f_{1}$ is supported on $U$ and $U \in$ E.

Now let $V$ be any element of $\&$, and let $f$ be any homeomorphism supported on $V$. We show that $f \in G$. There exists an $f_{2} \in H$ such that $f_{2}(\bar{V}) \subseteq W_{0}$, since $V \in \mathfrak{E}$. Let $g_{1}=f_{1} g^{-1} f_{1}^{-1} g$. Now $g_{1} \in G$, since $g_{1}$ is the product of $g$ by a conjugate of $g^{-1}$, and $G$ is normal in $H$. We note that $g_{1}$ is $f_{1}$ on $U$ and a copy of $f_{1}^{-1}$ on $g^{-1}(U)$.

Let $f_{0}=f_{2} f f_{2}^{-1}$. Then $f_{0}$ is a copy of $f$ and is supported on $W_{0}$. If $f_{0} \in G$ then any conjugate of it is also an element of $G$, and thus $f \in G$. We show that $f_{0} \in G$. Let

$$
\begin{aligned}
h(x) & =f_{0}(x), & & x \in W_{0}, \\
& =g_{1}^{n} f_{0} g_{1}^{-n}(x), & & x \in g_{1}^{n}\left(W_{0}\right), \quad n \geqq 1, \\
& =x, & & x \in X-\bigcup_{i=0}^{\infty} W_{i} .
\end{aligned}
$$

Then it is easy to see that $f_{0}=g_{1} h^{-1} g_{1}^{-1} h$, which is a product of $g_{1}$ by a conjugate of $g_{1}^{-1}$. Since $g_{1} \in G$, it follows that $f_{0}$, and therefore $f$, is also in $G$.

Corollary 3.1.1. Let $\{X, A, \mathfrak{B}, H\}$ be a s-l-s-h structure, and let $\mathfrak{F}_{1}$ and $\mathfrak{F}_{2}$ be near bases for $X$. Let $Q\left(\mathfrak{F}_{i}\right)$ be the basic subgroups of $H(X)$ generated by $\mathfrak{F}_{i}, i=1,2$. Then $Q\left(\xi_{1}\right)=Q\left(\xi_{2}\right)$.

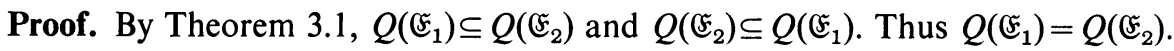

REMARK. It follows from the corollary that $Q(\mathfrak{E})$ is independent of $\mathbb{E}$, and therefore is unique. We denote the minimal normal subgroup by $Q(X)$ and call it the basic subgroup of $H$. We note that in the case of a compact, connected manifold, our $Q(X)$ coincides with the group $Q(X)$ defined in [2].

4. Additional properties of l-s-h and s-l-s-h continua. In this section we study the core of $X, M(X)$. Recall (from the Remark following Theorem 2.2) that $M(X)$ is defined to be the union of the elements of $\mathfrak{E}$, where $\mathbb{E}$ is a near basis for $X$. From Theorem 2.2 it follows that all near bases produce the same set.

TheOREM 4.1. Let $\{X, A, \mathfrak{B}, H\}$ be a l-s-h structure, and let $x \in X$. Then if $O(x)$ is not dense, it is nowhere dense.

Proof. Suppose there exists an open set $U$ such that $[O(x)]^{-} \cap U=U$. Let $V$ be an open set such that $\bar{V} \cap[O(x)]^{-}=\varnothing$. Let $y \in A \cap[O(x)]^{-}$. Then it follows (Lemma 2.3 of [1]) that there exists $h \in H$ such that $h(y) \in V$. Since $h$ is continuous, there exists a neighborhood $W$ of $y$ such that $h(W) \subseteq V$. Now $W$ must contain some point of $O(x)$, say $z$. Then there exists a homeomorphism $g \in H$ such that $g(x)=z$. 
Then $h g(x)=h(g(x))=h(z) \in h(W) \subseteq V$. Thus $h g$ is a homeomorphism taking $x$ into $V$. Therefore $\bar{V} \cap[O(x)]^{-} \neq \varnothing$. This is a contradiction. It follows that $O(x)$ is nowhere dense.

TheOREM 4.2. Let $\{X, A, \mathfrak{B}, H\}$ be a $s$-l-s-h structure. Then

(1) $M(X)$ is an open dense subset of $X$,

(2) $M(X)$ is the union of the dense orbits of $X$, and

(3) $M(X)$ is connected.

Proof. (1) Let $\&$ be a near basis for $X$. Then $M(X)$ is the union of the elements of $\&$ and is therefore open. Since $\&$ is a near basis for $X, M(X)$ contains $A$, and is therefore dense.

(2) Let $x \in M(X)$ and let $U$ be open in $X$. Let $\mathbb{E}=\{h(B)$, for some $B \in \mathfrak{B}$ and all $h \in H$ \} be a near basis for $X$. We first show that there exists an $h \in H$ such that $h(x) \in U$.

Since $x \in M(X)$, there exists an $f \in H$ such that $x \in f(B)$. Since $\&$ is a near basis for $X$, there is a $g \in H$ such that $g(B) \subseteq U$. Let $h=g f^{-1}$. Then $h(x) \in U$. Thus, if $x \in M(X)$, then $O(x)$ is dense. We next show that $O(x) \subseteq M(X)$. Let $y \in O(x)$. Now there is a homeomorphism $f \in H$ such that $x \in f(B)$. Also there is a $\phi \in H$ such that $\phi(x)=y$. It follows that $\phi f \in H$ and $\phi f(B) \subseteq M(X)$. But $y \in \phi f(B)$. Thus $y \in M(X)$. So we have now shown that if $x \in M(X)$ then $O(x)$ is dense and $O(x) \subseteq M(X)$.

It remains to be shown that if $O(x)$ is dense then $O(x) \subseteq M(X)$. From the above, it is sufficient to show that $x \in M(X)$. Let $E \in \mathbb{E}$, a near basis for $X$. Since $O(x)$ is dense, there is a point $y \in E$ such that $y=h(x)$ for some $h \in H$. From the above, $O(y) \subseteq M(X)$. But $x=h^{-1}(y) \in O(y)$. Thus $x \in M(X)$ and $O(x) \subseteq M(X)$.

It follows that $M(X)$ is the union of the dense orbits of $X$.

(3) Suppose $M(X)=P \cup Q$, where $P, Q$ are mutually separate sets. Since $M(X)$ is open and $P$ and $Q$ are open in $M(X), P$ and $Q$ are open in $X$. Let $a \in A \cap P$, $b \in A \cap Q$. Let $\varepsilon=\min \{d(a, C(P)), d(b, C(Q))\}$. Let $\mathscr{C}: C_{1}, C_{2}, \ldots, C_{n}$ be a chain of elements of $\mathscr{B}$ from $a$ to $b$ such that $\mu(\mathscr{C})<\varepsilon / 2$. Let $h$ be a homeomorphism of $X$ onto itself, $h$ supported on $\mathscr{C}^{*}$, such that $h(a)=b$. (Clearly $h$ exists for $h$ may be chosen to be $h_{n} \ldots h_{2} h_{1}$, where $h_{i}$ is supported on $C_{i}$ and carries $h_{i-1} \ldots h_{1}(a)$ to a point of $C_{i} \cap C_{i+1}$ for $2 \leqq i \leqq n-1$, and $h_{1}(a) \in C_{1} \cap C_{2}$, and $h_{n}$ carries $h_{n-1} \ldots h_{2} h_{1}(a)$ to $b$.) Now $\mathscr{C}^{*}$ is connected, and $X-M(X)$ separates $\mathscr{C}^{*}$. Let $D=[X-M(X)] \cap \mathscr{C}^{*}$. Then $D$ separates $a$ from $b$ in $\mathscr{C}^{*}$. Thus $h(D)$ separates $h(a)$ from $h(b)$ in $\mathscr{C}^{*}$, and therefore in $C_{n}$. But $C_{n} \subseteq M(X)$ and therefore cannot contain any points of $h(D)$. For if $x \in C_{n} \cap h(D)$ then $x \in M(X)$, and since $O(x) \subseteq M(X)$, $h^{-1}(x) \in M(X)$. But $h^{-1}(x) \in D$ and $D \cap M(X)=\varnothing$. This is a contradiction.

It follows that $M(X)$ is connected.

REMARK. The following question seems natural. Suppose $\{X, A, \mathscr{B}, G\}$ is a l-s-h structure, where $G$ is a proper subgroup of $H$. Suppose also that there exists a $B \in \mathscr{B}$ such that for each open set $U$ in $X$, there exists a $g \in G$ with $g(B) \subseteq U$. Let $\mathfrak{S}=\{g(B) \mid g \in G\}$. Let $M(\mathfrak{S})$ be the union of the elements of $\mathfrak{S}$. Is $M(\mathfrak{S})$ necessarily 
the same as $M(X)$ ? The answer is "No", for the following reason. Let $X$ be the universal plane curve, and let $\{X, A, \mathscr{B}, H\}$ be a l-s-h structure for $X$. Let $\varepsilon=$ diameter of $C$, where $C$ is the outer complementary bounding simple closed curve and let $\mathscr{B}^{\prime}=$ $\{B \in \mathscr{B} \mid$ diam $B<\varepsilon / 2\}$. Let $G$ be the subgroup of $H$ generated by those elements of $H$ which are supported on a single element of $\mathfrak{B}^{\prime}$. Then $\left\{X, A, \mathscr{B}^{\prime}, G\right\}$ is an l-s-h structure for $X$. Let $B_{0} \in \mathfrak{B}^{\prime}$ such that $\bar{B}_{0} \cap C=\varnothing$. Let $\mathbb{E}=\left\{g\left(B_{0}\right) \mid g \in G\right\}$. Then $M(\mathbb{E})$ cannot contain $C$, because each element of $G$ leaves $C$ setwise fixed. However, $M(X)=X$.

5. Continua which are l-s-h and s-l-s-h. It is known that the universal plane curve, universal curve, and the compact connected manifolds, with or without boundary, are l-s-h. (See [1] for a discussion.) From this it is clear that the following continuum is also l-s-h:

(This example was brought to my attention by R. D. Anderson.) Let $X$ be a compact two-manifold without boundary. Remove the interiors of a countable dense set of pairwise disjoint simple closed curves whose diameters have limit 0 . The resulting continua are clearly l-s-h.

In this section we show that the above mentioned examples, with the possible exception of the universal curve and Hilbert cube, are also s-l-s-h. In addition, we show that the Hilbert cube is l-s-h.

THEOREM 5.1. A compact manifold is $s-l-s-h$.

Proof. Let $X$ be a compact $n$-manifold, $A=$ Int $X, \mathfrak{B}$ be a basis of connected open sets whose closures are cells and such that for each $B \in \mathfrak{B},(\bar{B}-B)$ is a $(n-1)$ sphere and let $H$ be the group of homeomorphisms of $X$. Then it is easy to see that $\{X, A, \mathfrak{B}, H\}$ is a l-s-h structure for $X$. Now let $B \in \mathfrak{B}, x \in A \cap B$. Let $\phi: \bar{B} \rightarrow \rightarrow S$ be a homeomorphism of the cell $B$ onto the $n$-ball $S$, where $S=\left\{p \in R^{n} \mid d(p\right.$, origin) $\leqq 1\}$, and such that $\phi(x)=$ origin. Let $S^{\prime}$ be the $n$-ball of radius $\frac{1}{2}$, and let $U$ $=\phi^{-1}\left(\right.$ Int $\left.S^{\prime}\right)$. Then $\bar{U} \subseteq B$.

We show that $\bar{U}$ moves freely in $B$. Let $V$ be open in $B$. Then there exists a homeomorphism $h, h$ supported on $B$, such that $h(x) \in V$. Now $U \cap h^{-1}(V)$ is an open subset of $U$ containing $x$. Then $\phi\left(U \cap h^{-1}(V)\right)$ is an open subset of $S^{\prime}$ containing the origin. There exists a homeomorphism $\Psi: S \rightarrow S$ which keeps the origin fixed and carries $S^{\prime}$ into $\phi\left(U \cap h^{-1}(V)\right)$. Then $\phi^{-1} \Psi \phi$ is a homeomorphism of $X$ supported on $B$ and carrying $U$ into $U \cap h^{-1}(V)$. It follows that $h \phi^{-1} \Psi \phi$ is supported on $B$ and carries $U$ into $V$.

THEOREM 5.2. The universal plane curve is $s-l-s-h$.

Proof. Let $\{X, A, \mathfrak{B}, H\}$ be a l-s-h structure for the universal plane curve $X$. Let the outer bounding simple closed curve be denoted by $C_{0}$, and let the other complementary bounding simple closed curves be denoted by $\left\{C_{i}\right\}_{i=1}^{\infty}$. Let $C$ $=\bigcup_{i=0}^{\infty} C_{i}$. Let $B \in \mathfrak{B}, x \in A \cap B$. Let $U$ be a neighborhood of $x$ such that (1) $\bar{U} \subseteq B$ and (2) Bd $U$ is a simple closed curve not meeting $C$. Let $V$ be any open set 
in $B$ and let $y \in A \cap V$. Let $W$ be a neighborhood of $y$ such that (1) $\bar{W} \subseteq V$ and (2) $\mathrm{Bd} W$ is a simple closed curve not meeting $C$.

We show that there exists a homeomorphism $h, h$ supported on $B$, such that $h(\bar{U}) \subseteq V$. Let $N$ be an open set containing $\bar{U} \cup \bar{V}$ in its interior such that (1) $\bar{N} \subseteq B$ and (2) Bd $\bar{N}$ is a simple closed curve not meeting $C$. From the proof of Lemma 1 of [6], it follows that there exists a homeomorphism $\phi: \bar{N}-U \rightarrow \bar{N}-W$ taking $\mathrm{Bd} \bar{N} \rightarrow \operatorname{Bd} \bar{N}$ by the identity, and Bd $U$ onto Bd $W$. Further, the lemma just cited also shows that $\phi$ can be extended to take $\bar{U}$ onto $\bar{W}$.

COROLLARY 5.2.1. The example described at the beginning of this section is $s-l-s-h$.

Proof. Clear.

THEOREM 5.3. The Hilbert cube, $I^{\infty}$, is $l-s-h$.

Proof. Let $I^{\infty}=\prod_{i=1}^{\infty} I_{i}$, where $I_{i}=[0,1]$. Let $A=\prod_{i=1}^{\infty} I_{i}^{0}$, where $I_{i}^{0}=(0,1)$. Then $A$ is dense in $I^{\infty}$. Let $B$ be the collection of all connected open sets of $I^{\infty}$ of the form $U_{1} \times U_{2} \times \cdots \times U_{n} \times \prod_{i=n+1}^{\infty} I_{i}$, where $U_{i}$ is a connected open subset of $I_{i}$, in the topology of $I_{i}$. Then $\mathfrak{B}$ is a basis of connected open sets of $I^{\infty}$. Let $H$ be the group of all homeomorphisms of $I^{\infty}$ onto itself. We will show that $\left\{I^{\infty}, A, \mathfrak{B}, H\right\}$ is a l-s-h structure for $I^{\infty}$.

Let $B \in \mathfrak{B}$ with $B=U_{1} \times U_{2} \times \cdots \times U_{n} \times \prod_{i=n+1}^{\infty} I_{i}$. Let $a, b \in A \cap B$ with $a$ $=\left(a_{1}, a_{2}, \ldots\right), b=\left(b_{1}, b_{2}, \ldots\right)$. We will define a homeomorphism $h: I^{\infty} \rightarrow I^{\infty}$ such that $h(a)=b$. We obtain $h$ as an infinite product. Let $h_{i}^{\prime}: I_{i} \rightarrow I_{i}$ be a homeomorphism such that (1) $h_{i}^{\prime}$ is supported on $(0,1) \cap U_{i}$ and (2) $h_{i}^{\prime}\left(a_{i}\right)=b_{i}$. Let $h_{i}$ be an extension of $h_{i}^{\prime}$ to all $I^{\infty}$ defined as follows. If $x=\left(x_{1}, x_{2}, \ldots, x_{i-1}, x_{i}, x_{i+1}, \ldots\right)$ then $h_{i}(x)=\left(x_{1}, x_{2}, \ldots, x_{i-1}, h_{i}^{\prime}\left(x_{i}\right), x_{i+1}, \ldots\right)$. Let $h=\prod_{i=1}^{\infty} h_{i}$. Then $h$ is a homeomorphism of $I^{\infty}$ onto itself, $h$ is supported on $B$, and $h(a)=b$.

NoTE. In a later paper, we hope to prove or give reference to theorems showing that the Hilbert cube and universal curve are also s-l-s-h.

\section{REFERENCES}

1. B. L. Brechner, On the dimensions of certain spaces of homeomorphisms, Trans. Amer. Math. Soc. 121 (1966), 516-548. MR 32 \#4662.

2. J. V. Whittaker, On isomorphic groups and homeomorphic spaces, Ann. of Math. (2) 78 (1963), 74-91. MR 27 \#737.

3. R. D. Anderson, The algebraic simplicity of certain groups of homeomorphisms, Amer. J. Math. 80 (1958), 955-963. MR $20 \# 4607$.

4. - One-dimensional continuous curves and a homogeneity theorem, Ann. of Math. (2) 68 (1958), 1-16. MR 20 \#2676.

5. ——, On topological infinite deficiency, Michigan Math. J. 14 (1967), 365-383. MR 35 \#4893.

6. G. T. Whyburn, Topological characterization of the Sierpiniski curve, Fund. Math. 45 (1958), 320-324. MR 20 \#6077.

7. S. Ulam and J. von Neumann, On the group of homeomorphisms of the surface of a sphere, Bull. Amer. Math. Soc. 53 (1947), 506. Abstract \#283.

UNIVERSITY OF FLORIDA, GAINESVILLE, FLoRIDA 32601 